\title{
Irresolute-Topological Groups
}

\author{
Moiz ud Din Khan, Afra Siab and Luubiša D.R. KoČınaC ${ }^{1}$
}

\begin{abstract}
We introduce and study two classes of topologized groups, which are called here irresolute-topological and Irr-topological, investigate their properties, and establish their differences from topological groups. For this purpose we employ irresoluteness of the group operations instead of continuity in the case of topological groups and their variations.
\end{abstract}

\section{INTRODUCTION}

If $(G, *)$ is a group, and $\tau$ a topology on $G$, then we say that $(G, *, \tau)$ is a topologized group. Given a topologized group $G$, a question arises about interactions and relations between algebraic and topological structures: which topological properties are satisfied by the multiplication mapping $m: G \times G \rightarrow G,(x, y) \mapsto x * y$, and the inverse mapping $i: G \rightarrow G$, $x \mapsto x^{-1}$. In the literature the most papers are related to the case of topological groups where group operations are continuous mappings. However, there are also many nice papers and interesting, deep results on other classes of topologized groups: paratopological (the multiplication mapping is jointly continuous), semitopological (the multiplication mapping is separately continuos), quasi-topological (paratopological groups in which the inverse mapping is continuous). In all these cases continuity play the crucial role. We refer the reader to the monograph [1] and the survey paper [11] where detail information about mentioned classes of topologized groups can be found, as well as references related to them.

In this article we do not require the group operations to be necessary continuous, but to satisfy some weaker forms of continuity. The first paper of this sort is the paper [2], where semi-continuity is used instead of continuity (see also [3] in connection with topologized groups). Here, we require the

2010 Mathematics Subject Classification. Primary 54H11; Secondary 22A05, 54C08, $54 \mathrm{H} 99$.

Key words and phrases. Semi-open set, semi-closed set, irresolute mapping, semihomeomorphism, semi-regular space, irresolute-topological group, Irr-topological group.

${ }^{1}$ The third author thanks the Higher Education Commission of Pakistan for financial support for his visit to the Mathematical Department of CIIT in January/February 2013, and the CIIT for hospitality. 
group operations to be irresolute mappings or their variations. Properties of such groups are investigated and compared with properties of topological groups.

\section{Definitions AND PRELiminaries}

Throughout this paper $(G, *, \tau)$, or simply $G$, will denote a group $(G, *)$ endowed with a topology $\tau$. The identity element of $G$ is denoted by $e$, or $e_{G}$ when it is necessary, the operation $*: G \times G,(x, y) \mapsto x * y$, is called the multiplication mapping and sometimes denoted by $m$, and the inverse mapping $G \rightarrow G, x \mapsto x^{-1}$ is denoted by $i$.

$X$ and $Y$ denote topological spaces on which no separation axioms are a priori assumed. For a subset $A$ of a space $X$ we denote by $\operatorname{Int}(A)$ and $\mathrm{Cl}(A)$ the interior and the closure of $A$, respectively. For a mapping $f: X \rightarrow Y$ from a space $X$ to a space $Y$ we write $f^{\leftarrow}(B)$ to denote the preimage of a set $B \subset Y$. Usual topological terminology and notation are used, as in [1] and [7].

In 1963, N. Levine [8] introduced the notion of semi-open sets and semicontinuous mappings, and in [5] Crossley and Hildebrand used semi-open sets to define irresolute mapping. These two (perhaps the most important) generalizations of continuity found a lot of applications in topology and have been studied in many papers. We define now these mappings and other notions we need in this article.

A subset $U$ od a topological space $X$ is said to be semi-open if there is an open set $V$ in $X$ such that $V \subset U \subset \mathrm{Cl}(V)$. If a semi-open set $U$ contains a point $x \in X$ we say that $U$ is a semi-open neighbourhood of $x$. The complement of a semi-open set is called semi-closed. By $\mathrm{SO}(X)$ we denote the set of all semi-open subsets of $X$. For some applications of semi-open sets see also [10].

It is known that the union of any family of semi-open sets is semi-open (while the intersection of two semi-open sets need not be semi-open), and that the product of two semi-open sets $A \subset X$ and $B \subset Y$ is semi-open in $X \times Y$ (see [8]).

A mapping $f: X \rightarrow Y$ between topological spaces $X$ and $Y$ is called:

(1) semi-continuous (resp. irresolute) if for each open (resp, semi-open) set $V \subset Y$ the set $f^{\leftarrow}(V)$ is semi-open in $X$. Evidently, the mapping $f$ is semi-open (irresolute) if for each $x \in X$ if for each open (semiopen) neighborhood $V$ of $f(x)$ there exists a semi-open neighborhood $U$ of $x$ such that $f(U) \subset V$;

(2) ([5]) pre-semi-open if for every semi-open set $A$ of $X, f(A)$ is semiopen in $Y$;

(3) ([5]) a semi-homeomorphism if $f$ is bijective, irresolute and pre-semiopen. 
Definition 2.1 ([4]). Let $(X, \tau)$ be a topological space and $A$ be a subset of $X$. Then $x \in X$ is called a semi-interior point (resp. semi-adherent point) of $A$ if there exist a semi-open set $U$ such that $x \in U \subset A$ (resp. each semi-open neighbourhood of $x$ meets $A$ ). The set of all semi-interior (semi-adherent) points of $A$ is called the semi-interior of $A$, denoted $\operatorname{sint}(A)$, (resp. the semi-closure of $A$, denoted $\mathrm{sCl}(A)$.

Lemma 2.1 ([5]). If $f: X \rightarrow Y$ is a semi-homeomorphism, then:

(1) $\mathrm{sCl}(f(A))=f(\mathrm{sCl}(A))$ for all $A \subset X$;

(2) $\operatorname{sint}(f(A))=f(\operatorname{sint}(A))$ for all $A \subset X$.

\section{IRRESOLUTE-TOPOLOGICAL GROUPS}

In this section we will introduce and investigate new classes of topologized groups which we call irresolute-topological and Irr-topological groups.

Definition 3.1. A topologized group $(G, *, \tau)$ is called an irresolute-topological group if for each $x, y \in G$ and each semi-open neighborhood $W$ of $x * y^{-1}$ in $G$ there exist semi-open neighborhoods $U$ of $x$ and $V$ of $y$ such that $U * V^{-1} \subset W$.

In what follows we need the following lemma, which is a consequence of a result from [2].

Lemma 3.1. If $(G, *, \tau)$ is an irresolute-topological group, then:

(1) $A \in \mathrm{SO}(G)$ if and only if $A^{-1} \in \mathrm{SO}(G)$;

(2) If $A \in \mathrm{SO}(G)$ and $B \subset G$, then $A * B$ and $B * A$ are both in $\mathrm{SO}(G)$.

Theorem 3.1. If $(G, *, \tau)$ is an irresolute-topological group, then the multiplication mapping $m: G \times G \rightarrow G$ and the inverse mapping $i: G \rightarrow G$ are irresolute.

Proof. Let $(x, y)$ be a point in $G \times G$ and let $W \subset G$ be a semi-open neighbourhood of $m(x, y)=x * y$. Since $G$ is an irresolute-topological group, and $W$ is a semi-open neighbourhood of $x *\left(y^{-1}\right)^{-1}$, there exist semi-open neighborhoods $U$ of $x$ and $V$ of $y^{-1}$ such that $U * V^{-1} \subset W$. From Lemma 3.1, $V^{-1}$ is a semi-open neighbourhood of $y$, and $U \times V^{-1}$ is a semi-open neighbourhood of $(x, y) \in G \times G$. Then $m\left(U \times V^{-1}\right) \subset W$ just means that $m$ is irresoute at $(x, y)$, and, as $(x, y)$ was an arbitrary point in $G \times G$, that $m$ is irresolute on $G \times G$.

To show that $i$ is irresolute, let $x \in G$ be a point and let $W$ be a semiopen neighbourhood of $i(x)=x^{-1}$. Then by Lemma 3.1, $W^{-1}$ is a semi-open neighbourhood of $x$ satisfying $i\left(W^{-1}\right)=W$, which means that $i$ is irresolute at $x$, hence on $G$.

The previous theorem and Examples 3.1 and 3.2 below suggest the introduction of the following class of groups, wider than the class of irresolutetopological groups and containing the groups from these two examples. 
Definition 3.2. A topologized group $(G, *, \tau)$ is said to be an Irr-topological group if both the multiplication mapping $m: G \times G \rightarrow G$ and the inverse mapping $i: G \rightarrow G$ are irresolute.

Because the composition of two irresolute mappings is irresolute, we have the following simple corollary.

Corollary 3.1. If $G$ is an Irr-topological group, then the mapping $f: G \times$ $G \rightarrow G,(x, y) \mapsto x * y^{-1}$ is irresolute.

Example 3.1. Let $G=\left\{1, \omega, \omega^{2}\right\}$ be the group of the cube roots of unity $\left(\omega=e^{2 \pi i / 3}, \omega^{2}=e^{4 \pi i / 3}\right)$ with the usual multiplication $m=$. of complex numbers, and let $\tau=\{\emptyset,\{0\}, G\}$ be a topology on $G$. An easy verification shows that the multiplication mapping $m: G \times G \rightarrow G,(x, y) \mapsto x \cdot y$, and the inverse mapping $i: G \rightarrow G, x \mapsto x^{-1}$, are irresolute mappings (actually, $i$ is a homeomorphism). So, $(G, \cdot, \tau)$ is an Irr-topological group which is neither an irresolute-topological group (for the semi-open neighbourhood $W=\left\{1, \omega^{2}\right\}$ is an semi-open neighbourhood of $\omega \cdot\left(\omega^{2}\right)^{-1}=\omega^{2}$, but there are no semiopen neighbourhoods $U$ of $\omega$ and $V$ of $\omega^{2}$ satisfying the conditions from the definition of irresolute topological groups, nor a topological group.

Example 3.2. The set $G=\{1,3,5,7\}$ is a group under multiplication $m=\odot_{8}$, the multiplication modulo 8 . Let $\tau=\{\emptyset, G,\{1\},\{1,3,5\}\}$ be the topology on $G$. We prove that $m$ and $i$ are irresolute mappings. First,

$$
S O(G)=\{\emptyset, G,\{1\},\{1,3\},\{1,5\},\{1,7\},\{1,3,5\},\{1,3,7\},\{1,5,7\}\} .
$$

For any $V \in S O(G)$, the preimage $m^{\leftarrow}(V)$ contains the open set $\{(1,1)\} \subset$ $G \times G$ which is dense in $G \times G$, i.e. for each $V \in S O(V)$ we have $\{(0,0)\} \subset$ $m^{\leftarrow}(V) \subset C l\left(\{(0,0)\}\right.$. This means that $m^{\leftarrow}(V)$ is semi-open in $G \times G$, hence $m$ is irresolute.

On the other hand, for each $V \in S O(G)$, it holds $i^{-1}(V)=V$, which means that $i$ is an irresolute mapping. So, $\left(G, \odot_{8}, \tau\right)$ is an Irr-topological group which is not a topological group ( $m$ is not continuous, for instance, at $(3,3) \in G \times G)$.

Remark 3.1. It is known that the family of semi-open sets in a topological space need not be a topology. Note that the family $\mathrm{SO}(G)$ in Example 3.2 is a topology on $G$ different from $\tau$. From the definition of irresolute-topological groups we have the following

Observation. If $(G, *, \tau)$ is an irresolute-topological group such that the family $\mathrm{SO}(G)$ is a topology on $G$ with $\mathrm{SO}(G) \neq \tau$, then $(G, *, \mathrm{SO}(G))$ is a topological group.

However, the previous statement need not be true for Irr-topological groups. The group $G$ in Example 3.2 witnesses this fact, because $G$ with the 
new topology $\mathrm{SO}(G)$ is not a topological group: for the $\mathrm{SO}(G)$-neighbourhood $\{1\}$ of $3 \odot_{8} 3^{-1}=1$ there is no neighbourhood $V$ of 3 with $V \oplus_{8} V^{-1} \subset\{1\}$.

Remark 3.2. In topological groups the separation axioms $T_{0}, T_{1}$ and $T_{2}$ are equivalent, and each topological group satisfies the axiom of regularity. However, we have a different situation for irresolute-topological groups and Irr-topological groups. (For semi-separation axioms, similar to the usual separation axioms, see $[9,6]$.

The group $G$ in Example 3.2 is $T_{0}$, hence semi- $T_{0}$ Irr-topological group which is not semi- $T_{1}$. The same group is not semi-regular because for the closed set $\{3,5,7\}$ of $G$ and the point $1 \notin\{3,5,7\}$ there are no disjoint semi-open neighbourhoods.

Remark 3.3. We note that the notions of topological group and irresolutetopological group are independent of each other.

Theorem 3.2. Let $\left(G, *, \tau_{G}\right)$ be an irresolute-topological group and $\left(H, \circ, \tau_{H}\right)$ a topological group. If $f: G \rightarrow H$ is a homomorphism and semi-homeomorphism, then $H$ is also an irresolute-topological group.

Proof. Let $x$ and $y$ be any two points in $H$ and let $W \subset H$ be a semiopen neighbourhood of $x \circ y^{-1}$. Let $a=f^{-1}(x), b=f^{-1}(y)$. Since $f$ is an irresolute mapping the set $f^{-1}(W)$ is a semi-open neighbourhood of $a * b^{-1}$, and as $G$ is an irresolute-topological group there are semi-open neighbourhoods $A$ and $B$ of $a$ and $b$, respectively with $A * B^{-1} \subset f^{-1}(W)$. Pre-semi-openness of $f$ implies that the sets $U=f(A)$ and $V=f(B)$ are semi-open neighbourhoods of $x$ and $y$ that satisfy

$$
U \circ V^{-1}=f(A) \circ(f(B))^{-1}=f\left(A * B^{-1}\right) \subset W .
$$

This means that $\left(H, \circ, \tau_{H}\right)$ is an irresolute-topological group.

Theorem 3.3. Let $f: G \rightarrow H$ be a homomorphism, where $\left(G, *, \tau_{G}\right)$ and $\left(H, \circ, \tau_{H}\right)$ are irresolute topological groups. If $f$ is irresolute at the neutral element $e_{G}$ of $G$, then $f$ is irresolute on $G$.

Proof. Let $x \in G$ be an arbitrary element, and let $W$ be a semi-open neighbourhood of $y=f(x)$ in $H$. Since the left translations in $H$ are semihomeomorphisms, there is a semi-open neighbourhood $V$ of the neutral element $e_{H}$ of $H$ such that $\ell_{y}(V)=y \circ V \subset W$. From irresoluteness of $f$ at $e_{G}$ it follows the existence of a semi-open neighbourhood $U$ of $e_{G}$ such that $f(U) \subset V$. But $\ell_{x}: G \rightarrow G$ is a pre-semi-open mapping, so that the set $x * U$ is a semi-open neighbourhood of $x$, for which we have

$$
f(x * U)=f(x) \circ f(U)=y \circ f(U) \subset y \circ V \subset W .
$$

Hence $f$ is irresolute at the point $x$ of $G$, and since $x$ was an arbitrary element in $G, f$ is irresolute on $G$. 
Theorem 3.4. Every open subgroup $H$ of an irresolute-topological group $(G, *, \tau)$ is also an irresolute-topological group.

Proof. We prove that for each $x, y \in H$ and each semi-open neighbourhood $W \subset H$ of $x * y^{-1}$ there exist semi-open neighbourhoods $U \subset H$ of $x$ and $V \subset H$ of $y$ such that $U * V^{-1} \subset W$. Since $H$ is open, and $W$ semiopen in $G$, the set $W \cap H=W$ is a semi-open in $G$ and contains $x * y^{-1}$. Apply now the fact that $G$ is an iiresolute-topological group to find semiopen neighbourhoods $A$ of $x$ and $B$ of $y$ such that $A * B^{-1} \subset W$. The sets $U=A \cap H$ and $V=B \cap H$ are semi-open subsets of $H$ which contain $x$ and $y$ and satisfy $U * V^{-1} \subset A * B^{-1} \subset W$, which means that $H$ is an irresolute-topological group.

Theorem 3.5. A nonempty subgroup $H$ of an irresolute-topological group $G$ is semi-open if and only if its semi-interior is nonempty.

Proof. Assume $x \in \operatorname{sint}(H)$. There is a semi-open set $V$ such that $x \in$ $V \subset H$. This implies $x * V \subset H$. For every $y \in H$ we have $y * V=$ $y * x^{-1} * x * V \subset y * x^{-1} * H=H$. Since $y * V$ is semi-open we conclude that $H=\cup\{y * V: y \in H\}$ is semi-open as the union of semi-open sets.

The converse is trivial.

Theorem 3.6. Let $(G, *, \tau)$ be an irresolute-topological group. Then every open subgroup of $G$ is semi-closed in $G$.

Proof. Let $H$ be an open subgroup of $G$. Then every left coset $x * H$ of $H$ is semi-open because $\ell_{x}$ is a pre-semi-open mapping. Thus, $Y=\bigcup_{x \in G \backslash H} x * H$ is also semi-open as a union of semi-open sets. Then $H=G \backslash Y$ is semiclosed.

Theorem 3.7. Let $U$ be a symmetric semi-open neighbourhood of the identity $e$ in an irresolute-topological group $G$. Then the set $L=\bigcup_{n=1}^{\infty} U^{n}$ is a semi-open and semi-closed subgroup of $G$.

Proof. It is easy to see that $L$ is a subgroup of $G$ : if $x \in U^{p}, y \in U^{q}$ are elements from $L$, then $x * y \in U^{p+q} \subset L$; if $x \in L$, say $x \in U^{k}$, then $x^{-1} \in\left(U^{-1}\right)^{k}=U^{k} \subset L$. Because all translations in $G$ are pre-semi-open mappings, $U^{n}$ is semi-open for each $n \in \mathbb{N}$, and thus also $L$ is semi-open in $G$. By Theorem 3.6, $L$ is semi-closed.

Corollary 3.2. Let $G$ be an irresolute topological group, and $\mu_{e}$ the collection of all semi-open neighbourhoods of e. Then:

(1) for every $U \in \mu_{e}$, there is a $V \in \mu_{e}$ such that $V^{-1} \subset U$;

(2) for every $U \in \mu_{e}$ and every $x \in U$, there is $V \in \mu_{e}$ such that $x * V \subset U$.

Proof. (1)It follows from the fact that the inverse mapping $i$ is irresolute and $i(e)=e$. 
(2) This statement follows from the following: $\ell_{x}$ is a semi-homeomorphismm, and $\ell_{x}(e)=x$, so that there is a neighbourhood $V$ of $e$ such that $\ell_{x}(V) \subset U$, i.e. $x * V \subset U$.

Theorem 3.8. Let $G$ be an irresolute topological group, $A \subset G$ and $\mu_{e}$ the system of all semi-open neighbourhoods of e. Then $\operatorname{sCl}(A)=\cap\{A * U: U \in$ $\left.\mu_{e}\right\}$.

Proof. Let $x \in \operatorname{sCl}(A)$ and let $U$ be a semi-open neighborhood of $e$. The inverse mapping $i$ is irresolute, so that $i(e)=e$ implies the existence of a semi-open neighbourhood $V$ of $e$ such that $i(V)=V^{-1} \subset U$. From $x * V \cap A \neq \emptyset$, it follows that for some $v \in V$ and some $a \in A$ it holds $a=x * v$, i.e. $x=a * v^{-1} \in A * V^{-1} \subset A * U$. As $U$ was an arbitrary element from $\mu_{e}$ it follows $\operatorname{sCl}(A) \subset \cap\left\{A * U: U \in \mu_{e}\right\}$.

Conversely, let $x \notin \mathrm{sCl}(A)$. Then there exists $V \in \mu_{e}$ such that $x *$ $V \cap A=\emptyset$. Pick a semi-open neighbourhood $U$ of $e$ such that $U^{-1} \subset V$; the existence of such a set $U$ follows from the irresoluteness of the inverse mapping $i$ at $e$. Then $\left(x * U^{-1}\right) \cap A=\emptyset$, thus $x \notin A * U$. Therefore, $\cap\left\{A * U: U \in \mu_{e}\right\} \subset \operatorname{sCl}(A)$, and the theorem is proved.

Theorem 3.9. Let $A$ and $B$ be subsets of an irresolute-topological group $G$. Then:

(1) $\mathrm{sCl}(A) * \mathrm{sCl}(B) \subset \mathrm{sCl}(A * B)$;

(2) $(\mathrm{sCl}(A))^{-1}=\mathrm{sCl}\left(A^{-1}\right)$;

(3) $(\operatorname{sint}(A))^{-1}=\operatorname{sInt}\left(A^{-1}\right)$.

Proof. (1) Suppose that $x \in \mathrm{sCl}(A), y \in \mathrm{sCl}(B)$. Let $W$ be a semi-open neighbourhood of $x * y$. Then there are semi-open neighbourhoods $U$ and $V$ of $x$ and $y$ such that $U * V \subset W$. Since $x \in \operatorname{sCl}(A), y \in \operatorname{sCl}(B)$, there are $a \in A \cap U$ and $b \in B \cap V$. Then $a * b \in(A * B) \cap(U * V) \subset(A * B) \cap W$. This means $x * y \in \operatorname{sCl}(A * B)$, i.e. we have $\operatorname{sCl}(A) * \operatorname{sCl}(B) \subset \operatorname{sCl}(A * B)$.

(2) Since the inverse mapping $i: G \rightarrow G$ is a semi-homeomorphism, then by Lemma 2.1 we have

$$
\mathrm{sCl}(i(A))=\operatorname{sCl}\left(A^{-1}\right)=i(\mathrm{sCl}(A))=(\mathrm{sCl}(A))^{-1} .
$$

(3) Again we use the fact that the mapping $i$ is a semi-homeomorphism, which by Lemma 2.1 gives

$$
\operatorname{sInt}\left(A^{-1}\right)=(\operatorname{sint}(A))^{-1} .
$$

which completes the proof.

\section{REFERENCES}

[1] A.V. Arhangel'skii, M. Tkachenko, Topological Groups and Related Structures, Atlantis Studies in Mathematics, Vol. 1, Atlantis Press/World Scientific, AmsterdamParis, 2008.

[2] E. Bohn, J. Lee, Semi-topological groups, Amer. Math. Monthly 72 (1965), 996-998. 
[3] J. Cao, R. Drozdowski, Z. Piotrowski, Weak continuity properties of topologized groups, Czech. Math. J. 60 (2010), 133-148.

[4] S.G. Crossley, S.K. Hildebrand, Semi-closure, Texas J. Sci. 22 (1971), 99-112.

[5] S.G. Crossley, S.K. Hildebrand, Semi-topological properties, Fund. Math. 74 (1972), 233-254.

[6] C. Dorsett, Semi separation axioms and hyperspaces, Internat. J. Math. Math. Sci. 4 (1981), 445-450.

[7] R. Engelking, General Topology, 2nd edition, Heldermann-Verlag, Berlin, 1989.

[8] N. Levine, Semi-open sets and semi-continuity in topological spaces, Amer. Math. Monthly 70 (1963), 36-41.

[9] S.N. Maheshwari, R. Prasad, Some new separation axioms, Ann. Soc. Sci. Bruxelles 89 (1975), 395-402.

[10] T.M. Nour, A note on some applications of semi-open sets, Internat. J. Math. Math. Sci. 21 (1998), 205-207.

[11] M. Tkachenko, Paratopological and semitopological groups vs topological groups, In: K.P. Hart, J. van Mill, P. Simon (eds.), Recent Progress in General Topology III, Atlantis Press, 2014, pp. 803-859.

\author{
Moiz ud Din KHAN \\ Department of Mathematics \\ COMSATS Institute of Information TeChNOLOGY \\ Chack Shahzad \\ ISLAMABAD 44000 \\ PAKISTAN \\ E-mail address: moiz@comsats.edu.pk
}

\author{
Afra Siab \\ Department of Mathematics \\ COMSATS Institute of Information TeChnology \\ Chack Shahzad \\ ISLAMABAD 44000 \\ PAKISTAN \\ E-mail address: afrasiab8413@gmail.com
}

LJUBis̄a D.R. KỡINAC

UNIVERSITY OF NiŠ

Faculty Sciences and Mathematics

VIŠEGRADSKA 33

18000 NiS̆

SERBIA

E-mail address: 1kocinac@gmail.rs 